\title{
Identité et redécouverte de Rhithrogena nivata (Eaton, 1871) (Ephemeroptera, Heptageniidae)
}

\author{
M. Sartori 1 \\ A.G.B. Thomas 2
}

Mots clés : Insectes, Ephémeroptera, Heptageniidae, lectotype, topotype, redescription.

La recherche de topotypes de Rhithrogena nivata (Eaton, 1871) a permis de retrowver cette espèce pour la première fois depuis sa description. Des imagos $\sigma$ et $\vartheta$ ont été obtenues par élevage de larves. Les genitalia des $\sigma$ sont identiques à ceux du seul exemplaire restant de la série type (lectotype, British Museum). Les imagos sont redécrites.

Rediscovery and redescription of Rhlthrogena nlvata (Eaton, 1871). (Ephemeroptera, Heptageniidae).

Keywords : Ephemeroptera, Heptageniidae, lectotype, topotype, newdescription.

A search for topotypes of Rhithrogena nivata (Eaton, 1871) yielded the first specimens seen since the original description. Male and fermale imagos were obtained by rearing larvae. The male genitalia are identical with those of the sole remairing specimen of the original type series, a lectotype in the British Museum. The imagos are redescribed.

\section{1. - Introduction}

En 1871, l'espèce Heptageriu nivata a été décrite des Alpes par A.E. Eaton. En 1885, le même auteur a redécrit cette espèce à partir du même matériel et l'a classée dans le genre Rhithrogena, combinaison encore admise aujourd'hui. Deux localites typiques signalées : "The Barberine in the Val de Trient (about $3700 \mathrm{ft}$.) and Lac de Combal (5 $774 \mathrm{ft}$.) ".

Le nom spécifique nivata est ensuite complète. ment tombé dans l'oubli et n'a figuré de façon crédible dáns aucune liste faunistique.

En 1960, Kimmins a désigné comme lectotype le seul individu restant de la série type (une imago o ). La même année, Grandi a redécrit une espèce de la faune italienne sous le nom prudent de "Rhithrogena? nivata Eaton, 1871 ". Cette dernière espèce a plus tard été identifiée à $R h$. loyolaea Navás, vivant aussi dans les Pyrénées et très répandue dans les Alpes (Thomas 1970).

\footnotetext{
1. Musee Zoologique, Place Riponne 6, 1005 Lausanne, Suisse. Ce travail fatt partie d'une thèse de Doctorat.

2. Laboratoire d'Hydrobiologie. U.P.S. UA 695 du C.N.R.S., 118. route de Narbonne 31062 Toulouse Cedex, France.
}

A partir de ce moment, le lectotype reste donc la seule référence attribuable au nom spécifique de nivala.

\section{2. - Recherche de topotypes}

Durant l'été 1969, l'un de nous (A.T.) a tenté de récolter des topotypes. Le Lac de Combal n'ayant pu être localisé, les captures de larves et leur élevage ont été effectués uniquement sur la Barberine (carte 1). Tous les adultes émergés appartenaient à l'espèce Rhithrogena loyolaea Na vás. Il est indispensable de signaler l'édification - depuis Eaton - de deux barrages hydroélectriques, retenant le Lac du Vieux Emosson $(2050 \mathrm{~m})$ et le Lac de Barberine $(1890 \mathrm{~m})$, catuse d'importantes perturbations du cours d'eau. En 1983, nous sommes retournés à la Barberine : une nouvelle retenue - le Lac d'Emosson - recouvre l'emplacement de l'ancien Lac de Barberine par suite de l'édification d'un autre bar. rage $(1950 \mathrm{~m})$, très récent, beaucoup plus important que le précédent. Aucun représentant du genre Rhithrogena n'a pu être capturé. 
Cette même année, il a en revanche été possible de retrouver la seconde localité typique. Le Lac de Combal (carte 1) est une petite retenue naturelle peu profonde, située en Italie, dans le Val Veni ouvert sur le Val d'Aoste, d'où sa fréquente appellation sur les cartes récentes de "Lago di Veni ou Lago di Miage (du nom du glacier) ". Un géographe canto-

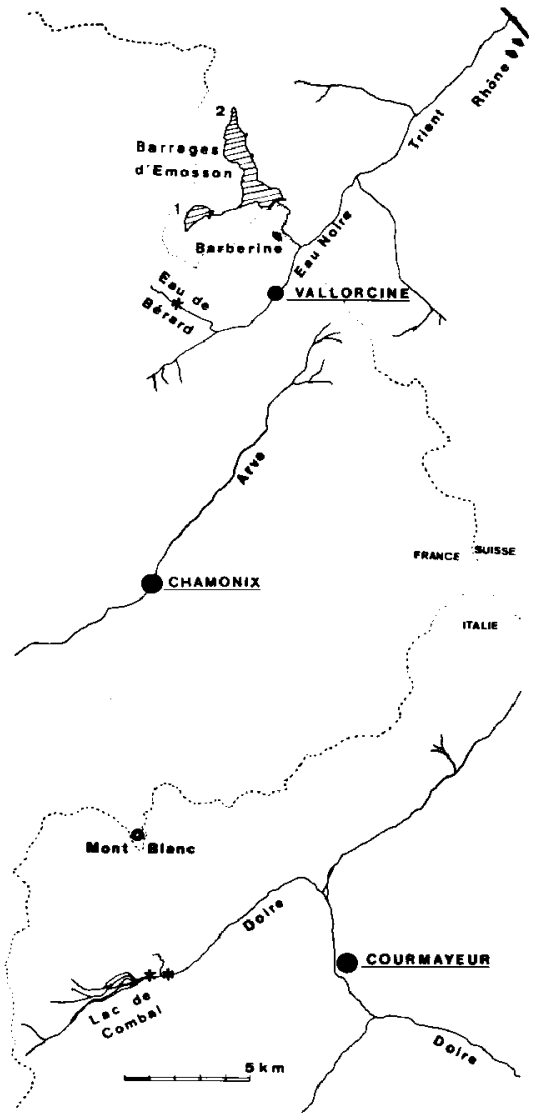

Carte 1. - Les stations de récolte $\left({ }^{\star}\right)$. nal nous a aimablement aidés à retrouver ce lac sous son ancienne appellation, confirmée par l'existence de vieux panneaux indicateurs routiers encore en place. Des prèlèvements effectués sur le déversoir du lac ont immédiatement révélé la présence d'une espèce de Rhithrogena à larve inconnue, très caractéristique par la forte pigmentation en arc cies branchies II à VI (fig. 1), visible à l'ceil nu. Les conditions de travail très défavorables (lit chaotique, très fort débit, circulation touristique intense, pollutions organiques) nous ont incité à rechercher dans les environs immédiats un cours d'eau comparable à la Barberine, mais peu ou pas perturbé par l'homme.

A moins de $5 \mathrm{~km}$ de la Barberine, se trouve l'Eau de Bérard. Ces cours d'eau sont tous deux des affluents de l'Eau Noire, principal tributaire du Irient. Ils coulent, avec une orientation voisine, sur le même versant. La largeur des lits est en outre comparable.

La prospection de l'Eau de Bérard nous a permis de constater rapidement la présence, en grand nombre, de larves de l'espece rencontrée au déversoir du Lac de Combal. La morphologie des genitalia o des imagos obtenues par élevage correspond bien aux figures de Kimmins (1960) relatives au lectotype de Rh. nivata. Elle correspond aussi aux autres dessins du type que cet auteur a aimablement effectués à notre intention en 1968 puis en 1970 . En outre, l'un de nous (M.S.) a eu la possibilité d'examiner récemment les genitalia de ce lectotype conservés en glycérine : ils sont semblables à ceux des spécimens de l'Eau de Bérard. Quant au dessin d'Eaton (1871 : fig. 10, pl. 10), il est trop sommaire pour permet tre une identification certaine de l'espece.

\section{3. - Redescription des imagos}

Diagnose sommaire : espèce de grande taille, brun moyen à foncé, pouvant présenter des maculations sombres autour des ganglions abdominaux; ailes entièrement hyalines.

Imago $\sigma$.

Tête.

Yeux de teinte rose chair à rougeât re testacé avec une bande latérale sousmarginale externe bleu noi. râtre qui précède une zone triangulaire gris blanchâtre. Face grisâtre réticulée de noir. Carène faciale brun moyen. Vertex brun foncé. Tubercule ocellaire largement de même couleur à la base, cer- 

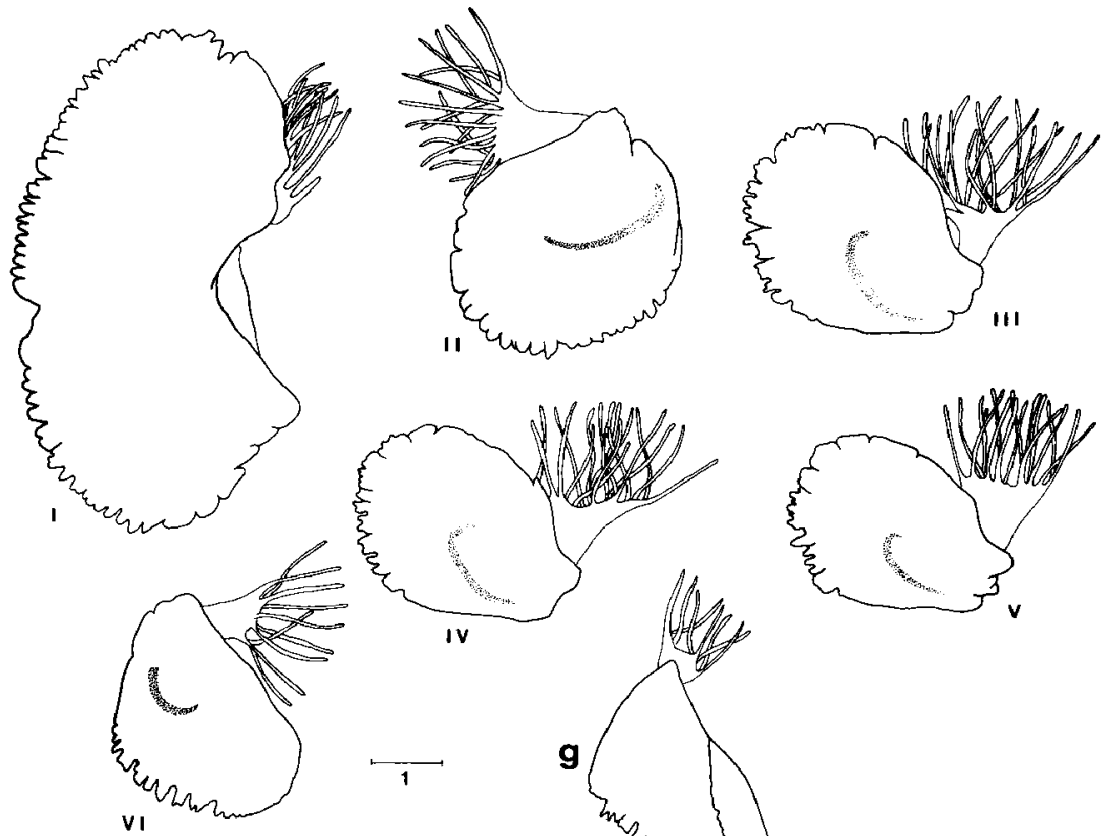

Fig. 1. Larve de Rhithrogena nivata Eaton, au dernier stade. Echelle en mm. Branchies droites ou gauches de la $I^{\circ}$ à la VII ${ }^{\circ}$ paires.

clé plus haut de brun moyen. Ocelles gris blanchâtre. Scape et pédicelle brun moyen. Funicule brun grisâtre, blanchâtre dans la région apicale.

\section{Thorax,}

Prothorax: pronotum brun grisâtre réticulé de noir. Stemite brun fonce. Coxa et trochanter I brun grisâtre. Fémur I brun moyen sale pouvant présenter une tache médiane peu marquée (1 individu sur les 4 examinés).

Meso et metathorax : mesonotum brun foncé brillant, scutellum et metanotum brun noirâtre. Pléures brun moyen à foncé. Membranes pleurales blanches. Sternites: mesothoracique brun foncé ; metathoracique plus clair, brun grisâtre. Pattes II et III : coxas brun grisâtre ; trochanters gris olivâtre avec l'extrémité de leur pointe apicale brun foncé: fémur's el tibias gris olivâtre avec une tache brun foncé à l'apex des fémurs; tarses brun sale. Les fémurs peuvent présenter ( 2 spécimens sur les 4) une tache longitudinale brun moyen, submédiane légèrement proximale (des variations peuvent être obser. vées d'un côté par rapport à l'autre). Aile antèrieure entièrement hyaline, à l'exception de l'aire ptérostigmatique, peu marquée, blanchâtre, portant de 12 à 15 nervures transverses peu ou pas ramifiées ; partout ailleurs, nervures longitudinales et transversales brun foncé. Aile postérieure hyaline à nervation brun grisâtre assez faible. 
Abdomen.

Tergites brun moyen grisâtre présentant un reticulum noirâtre ; membrane articulaire blanchâtre. Des traces de pigment brun moyen peuvent être visibles ou non dans la région des spiracles.

Sternites brun gris olivâtre, à ornementation variable. Les ganglions de la chaine abdominale peuvent être plus ou moins soulignés de pigment violacé noirâtre (sur un même individu certains ganglions peuvent être maculés, d'autres pas). L'intensité de la pigmentation est aussi très variable d'un individu à un autre. Partant du ganglion, deux traces obliques bistres, dirigées vers les pleures, sont parfois visibles. $9^{\mathrm{e}}$ sternite jaunâtre, brun près des pleures.

Cerques brun foncé assez uniforme, s'éclaircissant progressivement vers l'apex.

\section{Genitalia (fig. 2 à 6).}

Styles et styligère brun assez foncé. Incision médiane du styligère très largement ouverte, avec des bords presque rectilignes repoussant les deux protubérances latérales près de la base des styles (fig. 2). Lobes péniens eux aussi très écartés, avec une for te épine latérale externe - simple ou bifide - subapicale (fig. 3) et une grande dépression apicale plus ou moins ovale ( $/ \mathrm{ig} .4$ ). Près de cette dernière, une épine longitudinale (fig. 5 a \& b). Une petite épine surnuméraire peut se trouver implantée pres de l'une ou l'autre des deux précédentes ( $f \mathrm{ig}$. 4, 5 c). Titillateurs de taille moyenne, pluridentés (fig. 5,6 ), assez variables.

Taille.

Aile antérieure : $14 \mathrm{~mm}$; cerques : 26 à $29 \mathrm{~mm}$.

Imago $Q$.

Caractères distinctifs par rapport au $\sigma$ :

Coloration d'ensemble plus claire.

Vertex gris plus ou moins jaunátre tout autour du mamelon ocellaire.

Thorax brun jaunattre tout au plus brun moyen sur les sternites. Pattes gris olivâtre, foncé pour les antérieures, clair pour les $P$ II et P III. Tibia $I$ et tous les tarses assombris. Tache fémorale plus ou moins visible ou complètement absente.

Tergites abdominaux brun moyen, blanchâtres près des membranes articulaires et aussi, largement, dans la région postéro-inférieure. Sternites jaunâtres, plus ou moins olivátres. Les 7 spécimens examinés possèdent des ganglions abdominaux légè- rement maculés, à la périphèrie, d'un anneau de pigment violacé noirâtre. Traces sternales obliques en direction des pleures, bistres plus ou moins visibles.

Plaque sous-génitale brun foncé vers la périphérie et blanchâtre dans la région centrale et proximale.

Cerques brun grisâtre.

Taille.

Aile antérieure : 13,8 à $16 \mathrm{~mm}$; cerques : 13 à $20 \mathrm{~mm}$.

\section{Subimagos or et 9 .}

Vertex gris noirâtre.

Ailes antérieures uniformément gris moyen. Ailes postérieures gris clair à blanchâtre. Taches fémorales très variables en étendue et en intensité. Extrême pointe apicale des trochanters noire.

Taches périganglionnaires constantes sur le matériel examiné. Cerques brun grisâtre. Genitalia ơ (fig. 7) avec l'échancrure du styligère plus marquée, plus fermée que chez l'imago.

\section{4. - Matériel examiné}

Adultes : 4 o, 12 \%, 6 subimagos of et 6 \%, obtenus par élevage et provenant tous de l'Eau de Bérard à $1720 \mathrm{~m}$. (Haute-Savoie) aux dates suivantes: 17-22-VIII-1983 (1 o, 3 \&, 6 s. \%, 6 s. Q) et 15-IX-1983 (3 $\circ, 9$ \%).

Larves : plusieurs dizaines d'individus au dernier stade récoltés aux dates précitées à l'Eau de Bérard ; 17 exemplaires âgés capturés au déversoir du Lac de Combal entre 1800 et $1920 \mathrm{~m}$ - localité typique -, les 5 el 9-VIII-1983; 70 larves des collections A. Nadig et J Aubert. Ces dernières mont rent que $R h$. nivata est assez répandue sur le territoire helvétique: l'espèce est commune en particulier dans le Parc National Suisse.

Les individus sont conservés en alcool à $70^{\circ}$ et sont déposés au Musée Zoologique de Lausanne.

Remarque: la pigmentation mélanique périgan. glionnaire paraît variable, ce qui explique probablement le fait que les descriptions d'Eaton (1871, 1883-88) en fassent mention chez la $\$$ et pas chez le $\%$. 

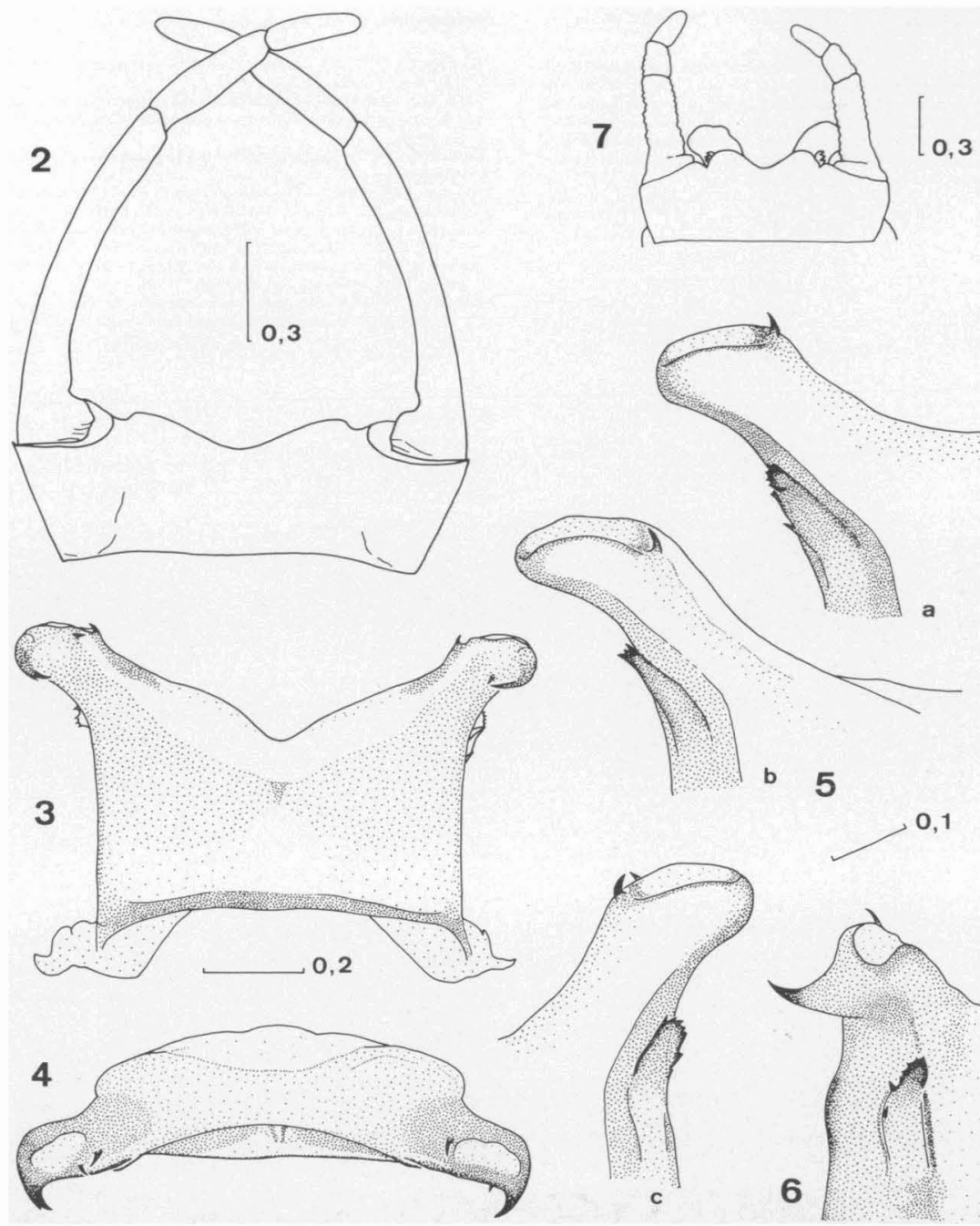

a

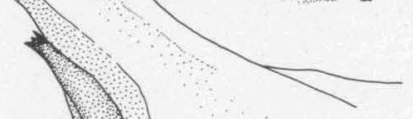

b 5
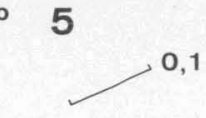

Fig. 2 à 7 . - Genitalia $O$ de Rhithrogena nivasta Eaton $(2$ a $6:$ imago: $7:$ subimago). Echelle en mm. 2 : styligère et styles en vue ventrale. 3 : pénis en vue dorsale. $4:$ pénis en vue apicale. 5 a, b et $c$ : apex de lobes péniens en vue ventrale, sous des angles légèrement différents. $6:$ lobe pénien vu de profil. $7:$ terminalix en vire ventrale. 


\section{Remerciements}

L'achevement de ce travail ar ele grandement facilite par l'accè aux collections Eaton et Aubert. C'est pour nous un plaisir de remercier MM. D.E. Kimmins et F. Barnard (British Museum, Lon. dres), J. Aubert, D. Cherix et P. Gueldlin (Musée Zoologique, Lausanne). Enfin, nous exprimons not re reconnaissance a $M . M$. Des. fayes (Fully) pour son aide a localiser la dernière station typique existante.
Travaux cites

Eaton (A.E.). 1871 - A monugraph on the Ephemeridac. Trans. $R$. ent. Soc., London : $1.164+6 \mathrm{pl}$.

Eaton (A.E.). 1883-88. - A revisional monograph of recent Ephemeridae or Mayflies. Trans. Linn. Soc. Lond., Zoology, $3: 1.352$ $+65 \mathrm{pl}$.

Grandi (M_). 1960 - Ephemeroidea. In ; Fauna d'Iralia, 3 ; 472 p., Bologna.

Kimmins (D.E.). 1960. - The Ephemeroptera tvpes of species described by A.E. Eaton, R. Mc Lachlan and F. Walker, with particular reference to those in the British Museum (Natural His. tory). Bull. Br. Mus. nat. Hist., Entomology, $9(4) ; 269-318$.

Puthz(V.). 1978. - Ephemeruptera. In J. Illies : Limnojauna Euno pdea. Fischer Verlag. Stutigarl, pp. 256-263.

Thomas (A.) 1970. $\rightarrow$ Sur la taxunomie de deux Rhithrogena des Pyrénées (Ephemeroptera, Heptageniidac). Annls Limnol., 6 (3) : 305.315 